\title{
Superconductor-metal quantum transition at the $\mathrm{EuO} / \mathrm{KTaO}_{3}$
}

\section{interface}

Yang $\mathrm{Ma}^{1 \dagger}$, Jiasen $\mathrm{Niu}^{1 \dagger}$, Wenyu Xing ${ }^{1}$, Yunyan $\mathrm{Yao}^{1}$, Ranran Cai ${ }^{1}$, Jirong Sun ${ }^{2,3}$, X. C. Xie ${ }^{1,4,5}$, Xi $\operatorname{Lin}^{1,4,5 *}$, Wei $\operatorname{Han}^{1 *}$

${ }^{1}$ International Center for Quantum Materials, School of Physics, Peking University, Beijing 100871, P. R. China

${ }^{2}$ Beijing National Laboratory for Condensed Matter Physics \& Institute of Physics, Chinese Academy of Sciences, Beijing 100190, P. R. China

${ }^{3}$ School of Physical Sciences, University of Chinese Academy of Sciences, Beijing 100049, P. R. China

${ }^{4}$ CAS Center for Excellence in Topological Quantum Computation, University of Chinese Academy of Sciences, Beijing 100190, P. R. China

${ }^{5}$ Beijing Academy of Quantum Information Sciences, Beijing 100193, P. R. China

†These authors contributed equally to the work

*Correspondence to: xilin@pku.edu.cn (X.L.) and weihan@pku.edu.cn (W.H.)

PACS: 74.78.-w, 73.40.-c, 73.43.Nq

\begin{abstract}
:
Superconductivity has been one of the most fascinating quantum states of matter for over several decades. Among the superconducting materials, $\mathrm{LaAlO}_{3} / \mathrm{SrTiO}_{3}$ interface is of particularly interest since superconductivity exists between two insulating materials, which provides it with various unique applications compared with bulk superconductors and makes it a suitable platform to study the quantum Hall effect, charge density wave, superconductivity and
\end{abstract}


magnetism in one device. Therefore, a lot of efforts have been made to search new superconducting oxide interface states with higher superconducting critical temperature $\left(T_{\mathrm{C}}\right)$. Recently, a superconducting state with $T_{\mathrm{C}} \sim 2 \mathrm{~K}$ has been found at the interface between a ferromagnetic insulator $\mathrm{EuO}$ and a band insulator (111)- $\mathrm{KTaO}_{3}$. Here, we report the experimental investigation of the superconductor-metal quantum phase transition of the $\mathrm{EuO} / \mathrm{KTaO}_{3}$ interface. Around the transition, a divergence of the dynamical critical exponent is observed, which supports the quantum Griffiths singularity in the $\mathrm{EuO} / \mathrm{KTaO}_{3}$ interface. The quantum Griffiths singularity could be attributed to large rare superconducting regions and quenched disorders at the interface. Our results could pave the way for studying the exotic superconducting properties at the $\mathrm{EuO} / \mathrm{KTaO}_{3}$ interface.

\section{INTRODUCTION}

Two-dimensional (2D) superconductivity at the $\mathrm{LaAlO}_{3} / \mathrm{SrTiO}_{3}$ interface has attracted a lot of attentions recently [1], which has exhibited interesting quantum phenomena, including the electrical-field-induced superconductor-insulator quantum phase transition [2] and coexistence of superconductivity with ferromagnetism [3-5]. Furthermore, $\mathrm{LaAlO}_{3} / \mathrm{SrTiO}_{3}$ interface might hold the promise towards future applications in the mesoscopic superconducting circuits [2]. Albeit its importance in fundamental physics and potential in applications, the extremely low $T_{\mathrm{C}}$ (below $300 \mathrm{mK}$ ) is a critical challenge [2,6]. Very recently, unexpected superconductivity is observed at the $\mathrm{EuO} /(111)-\mathrm{KTaO}_{3}$ interface which shows a $T_{\mathrm{C}}$ above $2 \mathrm{~K}$ [7]. The difference between 300 $\mathrm{mK}$ and $2 \mathrm{~K}$ is significant, because the former low temperature environment usually requires 
more expensive and rarer dilution refrigerators, while $2 \mathrm{~K}$ can be easily realized through evaporation of liquid helium or a close-cycle fridge simply based on electricity.

For 2D crystalline superconducting films/interfaces, the superconductor-metal/insulator phase transition is one of the most important properties [1]. Interestingly, in the case of quantum phase transitions involving a discrete symmetry breaking, i.e., when the system consists of large rare ordered regions, a quantum Griffiths singularity is theoretically expected to emerge [8,9]. Experimentally, the quantum Griffiths singularity has been observed in 2D superconducting Ga thin films [10], where the origin is theoretically discussed to be that the quenched disorder strongly influences the phase transition behavior and the resultant large rare superconducting regions are linked via long-range Josephson coupling. Subsequently, various low-dimensional superconducting systems have been shown to exhibit the quantum Griffiths singularity [11-16], albeit with detailed differences concerning the evolution of the dynamical critical exponent. Thus, testifying the quantum Griffiths singularity and the universality in a newly-discovered superconducting system is worth of more effort.

In this paper, we report the experimental investigation of superconductor-metal quantum phase transitions of the $\mathrm{EuO} /(111)-\mathrm{KTaO}_{3}$ interface. The superconductivity occurs at interface: the samples become as insulating as the pristine $\mathrm{KTaO}_{3}$ substrates after etching the EuO. In the Hall bar geometry, the $T_{\mathrm{C}}$ and $T_{\mathrm{BKT}}$ are determined to be $\sim 1.31 \mathrm{~K}$ and $\sim 1.42 \mathrm{~K}$, respectively, which are consistent with previous report utilizing the Van der Pauw geometry [7]. Around the superconductor-metal transition in the $\mathrm{EuO} / \mathrm{KTaO}_{3}$ interface, series of crossing points between neighboring magnetoresistance (MR) isotherms and the divergence of the dynamical critical exponent show up, which are features of quantum Griffiths singularity [10-16]. The quantum Griffiths singularity in the superconducting interface of the $\mathrm{EuO} / \mathrm{KTaO}_{3}$ heterostructures could 
arise from the quenched disorders that exist at the interface or the polycrystalline properties of the $\mathrm{EuO}$ layer.

\section{EXPERIMENTAL}

The $\mathrm{EuO} / \mathrm{KTaO}_{3}$ heterostructures were prepared by growing $\mathrm{EuO}$ thin films on the (111)$\mathrm{KTaO}_{3}$ substrates via oxide molecular beam epitaxy (MBE-Komponenten $\mathrm{GmbH}$; Octoplus 400). The (111)- $\mathrm{KTaO}_{3}$ substrates were ordered from Hefei Kejing Material Technology Co., Ltd. Prior to the $\mathrm{EuO}$ growth, the $\mathrm{KTaO}_{3}$ substrates were annealed at $500{ }^{\circ} \mathrm{C}$ in vacuum $\left(7 \times 10^{-10}\right.$ mbar) for $30 \mathrm{~min}$ to clean the surface. Then the $\mathrm{KTaO}_{3}$ substrates were kept at $500{ }^{\circ} \mathrm{C}$ during the growth of EuO thin films $(\sim 10 \mathrm{~nm})$, which included the following two steps. Firstly, a thin buffer layer of $\mathrm{Eu}(\sim 1 \mathrm{~nm})$ was grown onto the $\mathrm{KTaO}_{3}$ substrate using a thermal effusion cell. Then, the oxygen gas with a pressure of $1 \times 10^{-9}$ mbar was induced into the chamber, and the EuO of $\sim 9 \mathrm{~nm}$ was grown by reactive evaporation [17]. After the substrates were cooled down to $\sim 50$ ${ }^{\circ} \mathrm{C}$, a 5-nm MgO layer was deposited via $e$-beam evaporation before moving the samples out of the high-vacuum chamber. This thin $\mathrm{MgO}$ layer used to protect the EuO films from degradation during subsequent measurements.

The $\mathrm{EuO} / \mathrm{KTaO}_{3} \mathrm{Hall}$ bar devices were fabricated using standard photolithography and wetetching processes. Firstly, the $\mathrm{EuO} / \mathrm{KTaO}_{3}$ films were covered with photoresist by the spin coating process, and then were exposed to ultraviolet light through a photomask. Diluted hydrochloric acid was used to etch away the $\mathrm{EuO}$ to form the Hall bar geometry with a channel width of $100 \mu \mathrm{m}$ and a length of $4500 \mu \mathrm{m}$. The last step was to remove the residual chemicals on devices via acetone, IPA and DI water subsequently. 
For the electrical measurement from $T=300 \mathrm{~K}$ to $2 \mathrm{~K}$, the $\mathrm{EuO} / \mathrm{KTaO}_{3}$ heterostructures and Hall bar devices were measured in an Oxford Spectromag system with the d.c. technique $\left(I_{\mathrm{dc}} \sim\right.$ $100 \mu \mathrm{A})$. For the ultralow temperature measurement from $3.5 \mathrm{~K}$ to $71 \mathrm{mK}$, the resistance and magnetoresistance were measured in a dilution refrigerator (CF-CS81-600, Leiden Cryogenics BV) with the a.c. technique, using $10 \sim 100 \mathrm{nA}$ excitation current at $17 \mathrm{~Hz}$ and $2 \mathrm{mT} / \mathrm{s}$ (Fig. 2(b)) or $1 \mathrm{mT} / \mathrm{s}$ (Fig. 3) sweeping rate. Home-made resistor-capacitor (RC) filters and silver-epoxy filters were used in the dilution refrigerator to filter external high-frequency radiation and lower the electron temperature of samples, respectively. The electron temperature in this fridge has been shown to be equal to the refrigerator temperature above $25 \mathrm{mK}$ in previous fractional quantum Hall effect study [18].

\section{RESULTS}

Figures 1(a) and (b) show the RHEED patterns of the (111)- $\mathrm{KTaO}_{3}$ substrate and the polycrystalline EuO film (thickness: $\sim 10 \mathrm{~nm}$ ) viewed along [1-10] direction of $\mathrm{KTaO}_{3}$. The polycrystalline nature for $\mathrm{EuO}$ grown on (111) $-\mathrm{KTaO}_{3}$ substrates is due to the incompatible crystal structures and the large difference in in-plane lattice constant. The interface superconducting properties were characterized using the Van der Pauw (VdP) geometry (see Fig. $\mathrm{S} 1$ in supplementary materials) and Hall bar devices. For the VdP measurements with the current along the [11-2] and [1-10] directions, similar temperature dependences are observed, and $T_{\mathrm{C}}$ is about $1.33 \mathrm{~K}$ determined from the zero-resistance temperature, and the onset superconducting temperature $\left(T_{\mathrm{C}_{-} \text {onset }}\right)$ is around $1.90 \mathrm{~K}$.

The shcematics of the $\mathrm{EuO} /(111)-\mathrm{KTaO}_{3}$ Hall bar device are shown in Figure 1(c). After etching the $\mathrm{EuO}$ layer, the bare $\mathrm{KTaO}_{3}$ part of the $\mathrm{EuO} / \mathrm{KTaO}_{3}$ heterostructures become 
insulating, which further confirms the interface superconductivity. A typical optical image of the $\mathrm{EuO} / \mathrm{KTaO}_{3} \mathrm{Hall}$ bar device is shown in Fig. 1(d) inset. As the temperature decreases from 300 $\mathrm{K}$ to $1.5 \mathrm{~K}$, the mobility increases from $5 \mathrm{~cm}^{2} / \mathrm{Vs}$ to $98 \mathrm{~cm}^{2} / \mathrm{Vs}$, and the sheet carrier density decreases from $\sim 2.4 \times 10^{14} \mathrm{~cm}^{-2}$ to $\sim 7.4 \times 10^{13} \mathrm{~cm}^{-2}$ (supplementary Fig. S2). $T_{\mathrm{C}}$, defined as the zero-resistance temperature, is determined to be $\sim 1.31 \mathrm{~K}$ (Fig. 1(d)), which is almost the same as the $\mathrm{EuO} / \mathrm{KTaO}_{3}$ films characterized by Van der Pauw geometry.

Perpendicular magnetic field was applied to investigate the superconductor-metal transition of the $\mathrm{EuO} / \mathrm{KTaO}_{3}$ interface. As shown in Fig. 2(a), $T_{\mathrm{C}}$ of the $\mathrm{EuO} / \mathrm{KTaO}_{3}$ Hall bar device is strongly suppressed by the magnetic field. Under the magnetic field of $\sim 1.17 \mathrm{~T}$, zero-resistance state cannot be reached down to $T=71 \mathrm{mK}$, the lowest temperature of this measurement limited. When the magnetic field is further increased, the normal metallic state is induced. Based on the $B_{c \perp}$ vs. $T_{\mathrm{C}}$ curve (supplementary materials and Fig. S3), the critical perpendicular magnetic field is estimated to be $1.51 \mathrm{~T}$ at the absolute zero temperature, which corresponds to a superconducting coherence length of $\sim 14.8 \mathrm{~nm}$. These results are consistent with previous report with those values of $\sim 1.8 \mathrm{~T}$ and $13 \mathrm{~nm}$ at $\mathrm{T}=0 \mathrm{~K}$ [7]. Figure 2(b) shows the longitudinal and Hall resistances as functions of the perpendicular magnetic field at $T=91 \mathrm{mK}$. Clearly, a superconducting-to-normal-state transition happens at $B \sim 1.1 \mathrm{~T}$. Above this critical magnetic field, both the longitudinal resistance and Hall signal exhibit normal-metallic behavior. Based on the linear Hall resistance signal as a function of the magnetic field, the sheet carrier density is obtained to be $7.3 \times 10^{13} \mathrm{~cm}^{-2}$.

For 2D superconductors, Berezinskii-Kosterlitz-Thouless (BKT) transition characterizes the critical point where vortices and anti-vortices stabilize. When the temperature is slightly higher than $T_{\mathrm{BKT}}$ but lower than the $T_{\mathrm{C}_{-} \text {onset, }}$ the vortices and anti-vortices are mobile, which results in 
finite resistances [19-21]. To determine the BKT temperature $\left(T_{\mathrm{BKT}}\right)$, the current-voltage curves are presented in Fig. 2(c) in log-log plot. As the temperature increases, the critical current decreases dramatically (inset of Fig. 2(d)). When the applied current is larger than the critical current, the current-voltage curves merge, showing a linear behavior similar to the normal metallic state at $T=1.6 \mathrm{~K}$. The power-law scaling of $\mathrm{V} \propto I^{3}$ relationship is plotted (dashed purple line in Fig. 2(c)) to indicate the temperature around which the BKT transition happens [22]. To quantitatively determine $T_{\mathrm{BKT}}$, the current-voltage curves are fitted by the following equation,

$$
\mathrm{V} \propto I^{\alpha}
$$

where $\alpha$ is the power-law coefficient. As the temperature decreases, $\alpha$ continuously increases from 1 at $1.60 \mathrm{~K}$ to 10 at $1.38 \mathrm{~K}$ (Fig. $2(\mathrm{~d})$ ), from which $T_{\mathrm{BKT}}$ is determined to be $1.42 \mathrm{~K}$.

Figure 3 shows the magnetoresistance isotherms measured on the $\mathrm{EuO} / \mathrm{KTaO}_{3}$ Hall bar device at various temperatures from $1.00 \mathrm{~K}$ to $0.080 \mathrm{~K}$. Series of crossing points between neighboring MR isotherms are observed. As the temperature decreases, the magnetic field of the crossing points increases monotonically and a large enhancement of the critical magnetic field is observed at lower temperatures (inset of Fig. 3). The series of crossing points between MR isotherms are consistent with the quantum Griffiths singularity behaviors observed recently in 2D crystalline superconductors $[10-13,15,16]$.

\section{DISCUSSION}

To analyze this exotic phenomenon with series of crossing points, the finite-size scaling analysis is performed using the following formula [23-25], 


$$
R(B, T)=R_{\mathrm{c}} f\left[\left(B-B_{\mathrm{C}}\right) / T^{1 / z v}\right],
$$

where $R_{\mathrm{C}}$ is the critical resistance, $B_{\mathrm{C}}$ is the critical magnetic field, $f[]$ is an arbitrary function with $f[0]=1, z$ is the dynamic critical exponent, and $v$ is the coherence length exponent. For the purpose of effective analysis, the small crossing region with three adjacent $R(B)$ curves, is regarded approximately as one "critical" point. The Eq. (2) can be reformatted as $R\left(B_{\mathrm{c}}, \mathrm{t}\right) / R_{\mathrm{c}}=f$ $\left[\left(B-B_{\mathrm{c}}\right) t\right]$, where $t=\left(T / T_{0}\right)^{-1 / \mathrm{zv}}$ and $T_{0}$ is the lowest temperature in each group [11]. For the temperature range of $[0.8 \mathrm{~K}, 1.0 \mathrm{~K}]$, the critical values are $B_{\mathrm{C}}=1.483 \mathrm{~T}, R_{\mathrm{C}}=8263 \Omega, T_{0}=0.8$ $\mathrm{K}$. The finite-size scaling analysis of the MR isotherms is shown in fig. 4(a), and the data fall on to a bivalue curve. Similarly, figure 4(b) shows finite-size scaling analysis MR isotherms in the temperature range of $[0.080 \mathrm{~K}, 0.125 \mathrm{~K}]$ with $B_{\mathrm{C}}=1.717 \mathrm{~T}, R_{\mathrm{C}}=8617 \Omega$. The derived dynamical critical exponent $(z v)$ is obtained to be 0.77 in high temperature range $[0.80 \mathrm{~K}, 1.00$ K] (inset of Fig. 4(a)) and 2.6 in the lowest temperature range [0.080 K, $0.125 \mathrm{~K}]$ (inset of Fig. 4(b)). The accuracy of critical exponent is about $10 \%$. The systematical variation of $z v$ as a function of magnetic field for all the temperature ranges is summarized in Fig. 4(c). As the temperature decreases, $z v$ rapidly increases and exhibits a divergent behavior as a function of $B$. Quantitatively, this observation can be understood using the activated quantum scaling law $[26,27]$

$$
z v \approx \mathrm{C}\left|B-B_{c}^{*}\right|^{-v \psi},
$$

where $\mathrm{C}$ is a constant, $B_{c}^{*}$ is the derived critical magnetic field, and $v, \psi$ are the $2 \mathrm{D}$ infinite randomness critical exponents with $v \sim 1.2$, and $\psi \sim 0.5$ [26,27]. The activated quantum scaling curve (dashed line in Fig. 4c) well describes the observed experimental results, and $B_{c}^{*}$ is obtained to be $\sim 1.72 \mathrm{~T}$. 
The divergence of the dynamical critical exponent supports the quantum Griffiths singularity around the superconductor-metal transition, where singularity arises from the presence of large rare superconducting regions [28-30]. In this circumstance, the absence of long-range order does not necessarily blur the critical superconductor-metal quantum phase transition, but instead, the Griffiths singularity emerges due to the long-range Josephson coupling between separated superconducting islands, resulting global superconductivity [10]. It is also noted that recent theoretical studies [31,32] show that large rare superconducting regions are not sufficient for a true quantum Griffith singularity that is predicted by Fisher [8,9]. While experimentally, the superconductor to metal transitions in 2D Ga superconducting films exhibit the quantum Griffith behaviors [10], where the origin is theoretically attributed to the interplay of quenched disorder and thermal fluctuation. Our experimental results in $\mathrm{EuO} / \mathrm{KTaO}_{3}$ also exhibit the quantum Griffiths singularity feature around the superconductor-metal transition. Compared to previous reports, our observation is similar to quantum Griffith singularity reported in the 2D Ga superconducting films [10].

The observation of quantum Griffiths singularity in $\mathrm{EuO} / \mathrm{KTaO}_{3}$ indicates the possible quenched disorders that exist at the interface. Thus, the study of the correlation between the $T_{\mathrm{C}}$ and the quantum Griffith singularity might be important to reveal the disorder effect on $T_{\mathrm{C}}$ of the $\mathrm{EuO} / \mathrm{KTaO}_{3}$ interfaces. One of the possible sources of the quenched disorders could arise from the polycrystalline properties of the $\mathrm{EuO}$ layer, which is due to the large lattice mismatch between (111) $\mathrm{EuO}$ and (111)-faced $\mathrm{KTaO}_{3}$. By improving the quality of the $\mathrm{EuO}$ thin films to remove the quenched disorders at the interface, the quantum Griffith singularity feature might disappear. Future works are needed to identify to what extent the extrinsic disorder can affect the quantum Griffiths singularity and the $T_{\mathrm{C}}$ of the $\mathrm{EuO} / \mathrm{KTaO}_{3}$ interfaces. 


\section{CONCLUSION}

In summary, we have investigated the superconductor-metal transition properties of the interface superconductivity between a ferromagnetic insulator $\mathrm{EuO}$ and a band insulator $\mathrm{KTaO}_{3}$. With a Hall bar geometry, the $T_{\mathrm{C}}$ and $T_{\mathrm{BKT}}$ are determined to be $\sim 1.31 \mathrm{~K}$ and $\sim 1.42 \mathrm{~K}$, respectively, which are similar to the previous report of van der Pauw geometry measurement on the $\mathrm{EuO} / \mathrm{KTaO}_{3}$ interface [7]. Interestingly, the divergence of the dynamical critical exponent is observed as the temperature decreases, which supports the quantum Griffiths singularity in the $\mathrm{EuO} / \mathrm{KTaO}_{3}$ interface. The quantum Griffiths singularity could be attributed to large rare superconducting regions at the $\mathrm{EuO} / \mathrm{KTaO}_{3}$ interface. Our results bring motivation to further investigation of the exotic superconducting properties that could exist at the $\mathrm{EuO} / \mathrm{KTaO}_{3}$ interface, such as the coexistence of ferromagnetism and superconductivity, and unconventional spin-triplet superconductivity.

\section{ACKNOWLEDGMENTS}

This work is supported by the National Basic Research Programs of China (Nos. 2019YFA0308401 and 2017YFA0303301), National Natural Science Foundation of China ((Nos. 11974025, 11674009, 11934016, and 11921004), Beijing Natural Science Foundation (No. 1192009 and JQ18002), and the Key Research Program of the Chinese Academy of Sciences (Grant No. XDB2800000).

\section{References:}

[1] Y Saito, T Nojima, and Y Iwasa 2016 Nat. Rev. Mater. 216094 
[2] A D Caviglia, S Gariglio, N Reyren, D Jaccard, T Schneider, M Gabay, S Thiel, G Hammerl, J Mannhart, and J M Triscone 2008 Nature (London) 456624

[3] L Li, C Richter, J Mannhart, and R C Ashoori 2011 Nat. Phys. 7762

[4] J A Bert, B Kalisky, C Bell, M Kim, Y Hikita, H Y Hwang, and K A Moler 2011 Nat. Phys. 7767

[5] D A Dikin, M Mehta, C W Bark, C M Folkman, C B Eom, and V Chandrasekhar 2011 Phys. Rev. Lett. 107056802

[6] N Reyren, S Thiel, A D Caviglia, L F Kourkoutis, G Hammerl, C Richter, C W Schneider, T Kopp, A S Rüetschi, D Jaccard, M Gabay, D A Muller, J M Triscone, and J Mannhart 2007 Science 3171196

[7] X Y C. Liu, D. Jin, Y. Ma, H. Hsiao, Y. Lin, T. Sullivan, X. Zhou, J. Pearson, B. Fisher, J. Jiang, W.Han, J. Zuo, J. Wen, D. Fong, J. Sun, H. Zhou and A. Bhattacharya 2020 arXiv 2004 07416

[8] D S Fisher 1992 Phys. Rev. Lett. 69534

[9] D S Fisher 1995 Phys. Rev. B 516411

[10] Y Xing, H-M Zhang, H-L Fu, H Liu, Y Sun, J-P Peng, F Wang, X Lin, X-C Ma, Q-K Xue, J Wang, and X C Xie 2015 Science 350542

[11] S Shen, Y Xing, P Wang, H Liu, H Fu, Y Zhang, L He, X C Xie, X Lin, J Nie, and J Wang 2016 Phys. Rev. B 94144517

[12] Y Xing, K Zhao, P Shan, F Zheng, Y Zhang, H Fu, Y Liu, M Tian, C Xi, H Liu, J Feng, X Lin, S Ji, X Chen, Q-K Xue, and J Wang 2017 Nano Lett. 176802

[13] Y Saito, T Nojima, and Y Iwasa 2018 Nat. Commun. 9778

[14] E Zhang, J Zhi, Y-C Zou, Z Ye, L Ai, J Shi, C Huang, S Liu, Z Lin, X Zheng, N Kang, H Xu, W Wang, L He, J Zou, J Liu, Z Mao, and F Xiu 2018 Nat. Commun. 94656

[15] Y Liu, Z Wang, P Shan, Y Tang, C Liu, C Chen, Y Xing, Q Wang, H Liu, X Lin, X C Xie, and J Wang 2019 Nat. Commun. 103633

[16] C Zhang, Y Fan, Q Chen, T Wang, X Liu, Q Li, Y Yin, and X Li 2019 NPG Asia Mater. 1176

[17] Y Yun, Y Ma, T Su, W Xing, Y Chen, Y Yao, R Cai, W Yuan, and W Han 2018 Phys. Rev. Mater. 2034201

[18] P Wang, K Huang, J Sun, J Hu, H Fu, and X Lin 2019 Rev. Sci. Instrum. 90023905

[19] V L Berezinskii 1971 Sov. Phys. JETP 32493

[20] V L Berezinskii 1972 Sov. Phys. JETP 34610

[21] Kosterli.Jm and D J Thouless 1972 J. Phys. C 5124

[22] K Epstein, A M Goldman, and A M Kadin 1981 Phys. Rev. Lett. 47534

[23] S L Sondhi, S M Girvin, J P Carini, and D Shahar 1997 Rev. Mod. Phys. 69315

[24] A M Goldman 2010 Int. J. Mod. Phys. B 244081

[25] M P A Fisher 1990 Phys. Rev. Lett. 65923

[26] T Vojta, A Farquhar, and J Mast 2009 Phys. Rev. E 7901111

[27] I A Kovács and F Iglói 2010 Phys. Rev. B 82054437 
[28] N Markovic 2015 Science 350509

[29] T Vojta and J A Hoyos 2014 Phys. Rev. Lett. 112075702

[30] T Vojta 2006 J. Phys. A 39 R143

[31] B Spivak, P Oreto, and S A Kivelson 2008 Phys. Rev. B 77214523

[32] A Kapitulnik, S A Kivelson, and B Spivak 2019 Rev. Mod. Phys. 91011002

[33] C Richter, H Boschker, W Dietsche, E Fillis-Tsirakis, R Jany, F Loder, L F Kourkoutis, D A Muller, J R Kirtley, C W Schneider, and J Mannhart 2013 Nature (London) 502528

[34] B Kalisky, E M Spanton, H Noad, J R Kirtley, K C Nowack, C Bell, H K Sato, M Hosoda, Y Xie, Y Hikita, C Woltmann, G Pfanzelt, R Jany, C Richter, H Y Hwang, J Mannhart, and K A Moler 2013 Nat. Mater. 121091 
(a)

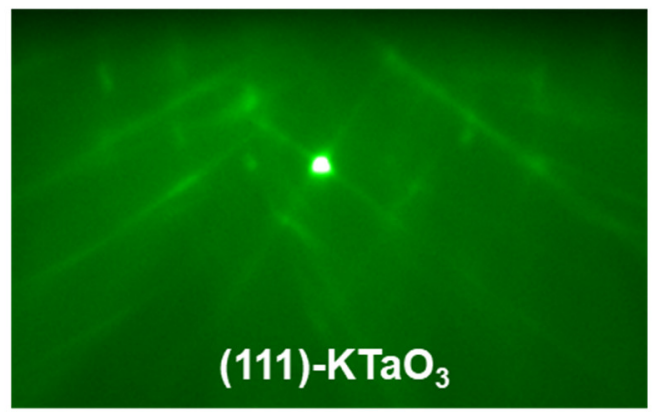

(b)

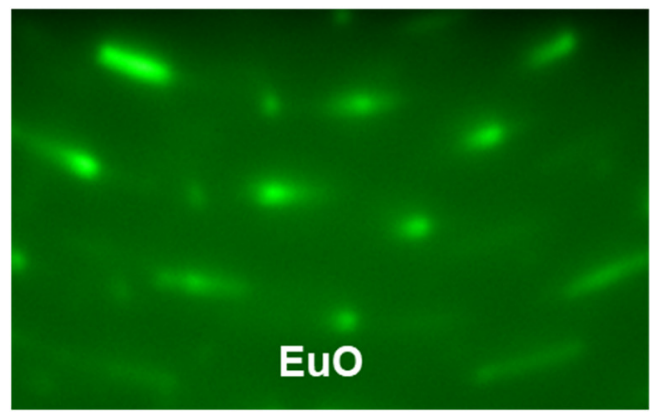

(c)

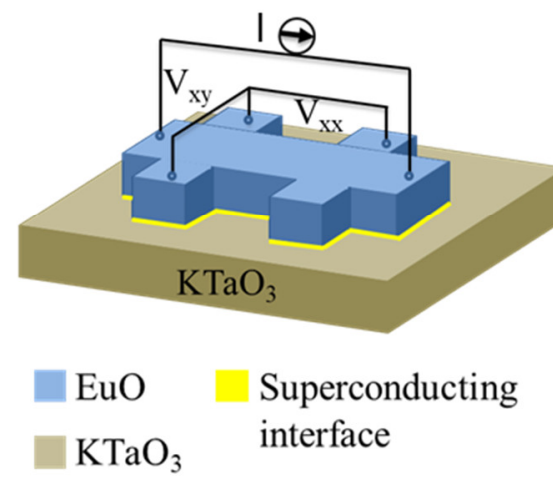

(d)

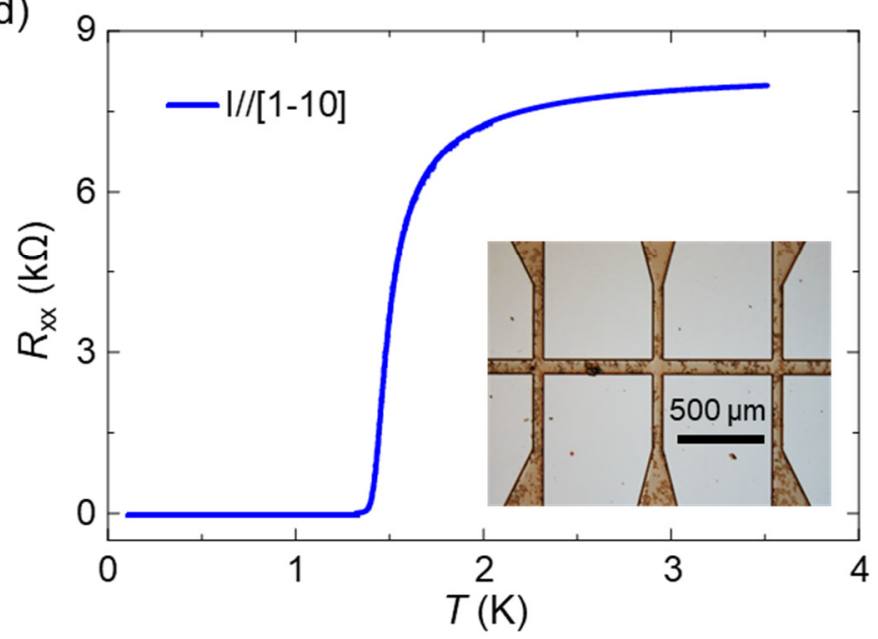

Figure 1. Growth and electrical measurement of the superconducting $\mathrm{EuO} /(111)-\mathrm{KTaO}_{3}$ interface. (a-b) RHEED patterns of a typical (111)-oriented $\mathrm{KTaO}_{3}$ substrate and the polycrystalline $\mathrm{EuO}$ thin film $(\sim 10 \mathrm{~nm})$ viewed from the $\mathrm{KTaO}_{3}$ crystal's [1-10] direction. (c) Schematic of the $\mathrm{EuO} / \mathrm{KTaO}_{3}$ Hall bar device and measurement geometry. (d) The longitudinal resistance $\left(R_{\mathrm{xx}}\right)$ as a function of temperature on the $\mathrm{EuO} / \mathrm{KTaO}_{3}$ Hall bar device. Inset: The optical image of the Hall bar device. 
(a)

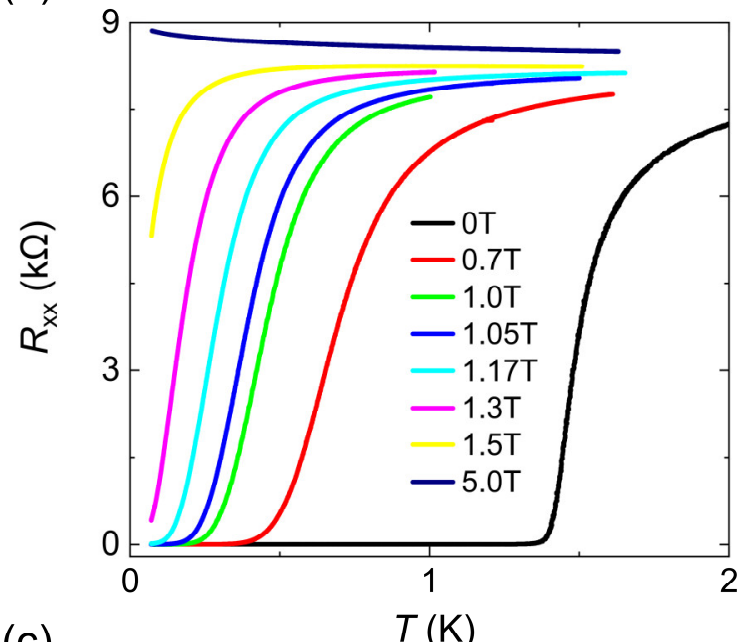

(c)

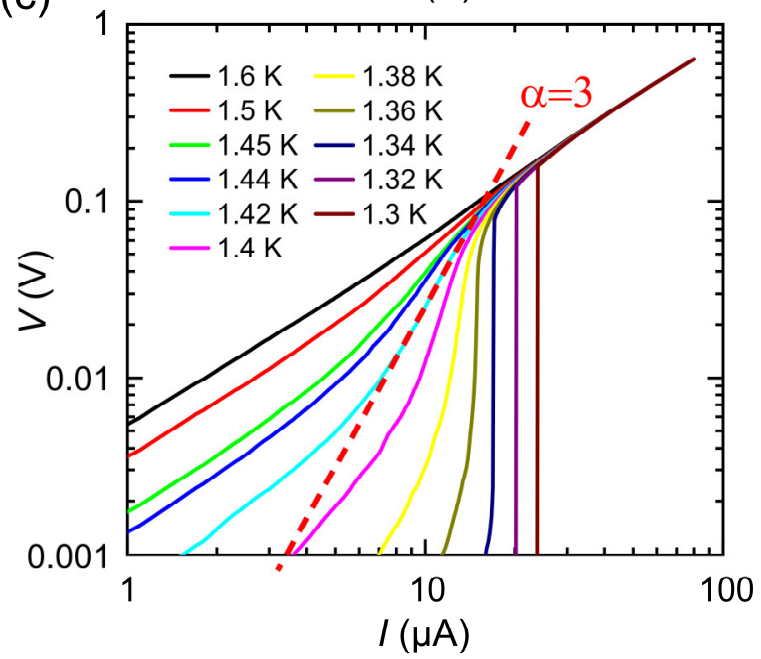

(b)

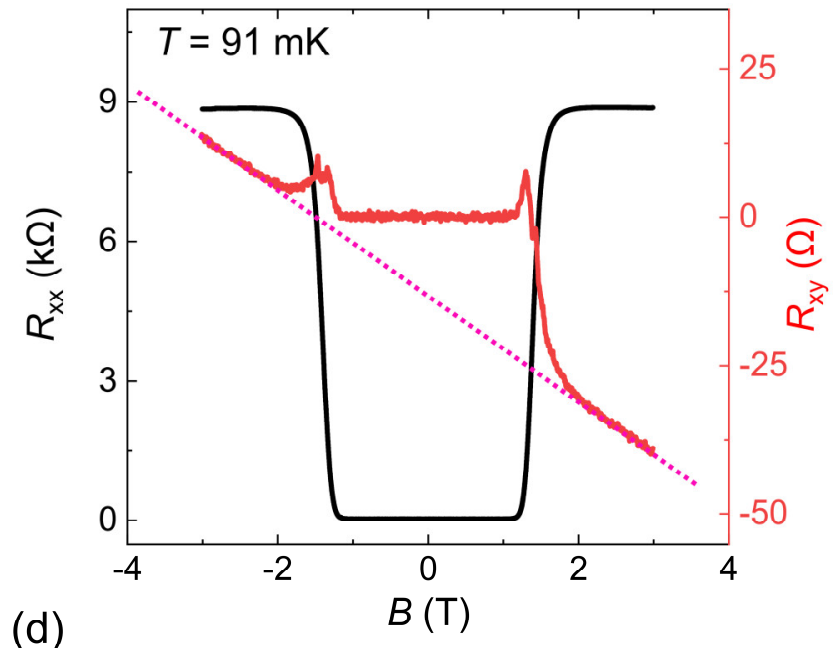

(d)

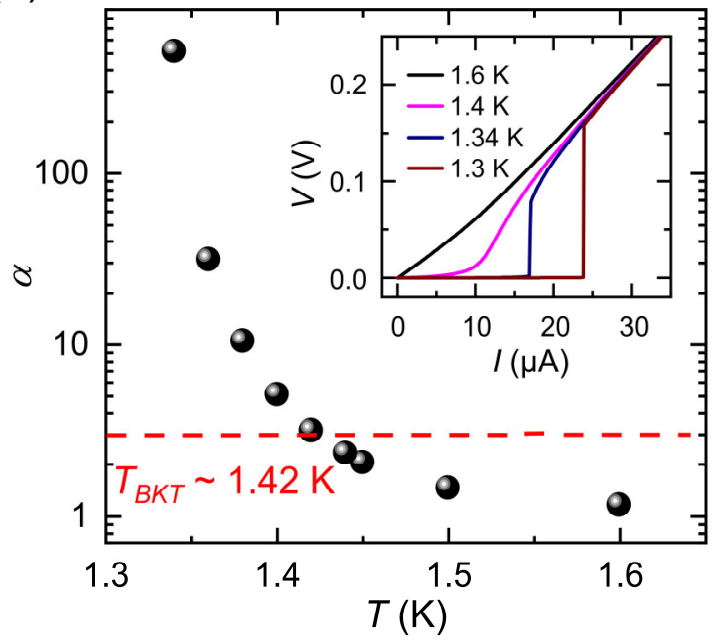

Figure 2. The BKT transition of the superconducting $\mathrm{EuO} /(111)-\mathrm{KTaO}_{3}$ interface. (a) The temperature dependence of the longitudinal resistance of the $\mathrm{EuO} / \mathrm{KTaO}_{3} \mathrm{Hall}$ bar device under various magnetic fields. The magnetic field is applied perpendicular to the interface. (b) The magnetic field dependence of the longitudinal and Hall resistances of the $\mathrm{EuO} / \mathrm{KTaO}_{3}$ Hall bar device at $T=91 \mathrm{mK}$. The purple dashed line represents the best linear fit for the Hall resistance at large magnetic fields. (c) The log-log curves of current-voltage dependence between $T=1.3$ and $1.6 \mathrm{~K}$. The red dashed line represents the relationship of $V \sim I^{3}$. (d) The BKT transition temperature $\left(T_{\mathrm{BKT}}\right)$ determined from the power coefficient $(\alpha)$ vs. $T$ plot. Inset: The currentvoltage curves at $T=1.3,1.34,1.4$, and $1.6 \mathrm{~K}$, respectively. 


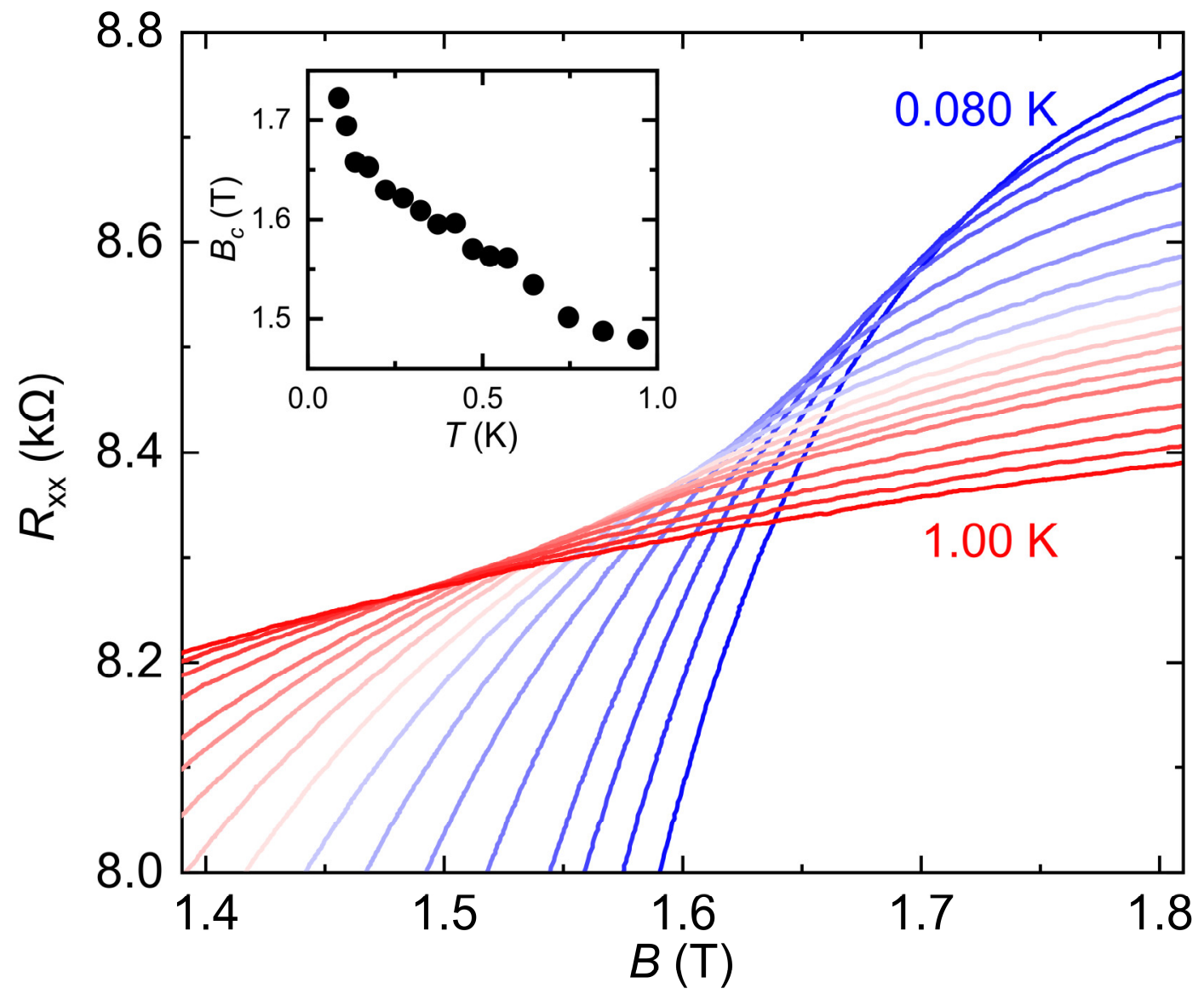

Figure 3. Magnetoresistance isotherms of the superconducting $\mathrm{EuO} / \mathrm{KTaO}_{3}$ interface. The magnetic field dependence of the longitudinal resistance of the $\mathrm{EuO} / \mathrm{KTaO}_{3}$ Hall bar device at various temperatures. Inset: The temperature dependence of the crossing magnetic field $\left(B_{c}\right)$ that could be extracted from $R_{\mathrm{xx}}$ Vs. $B$ curves at every two adjacent temperatures. 
(a)

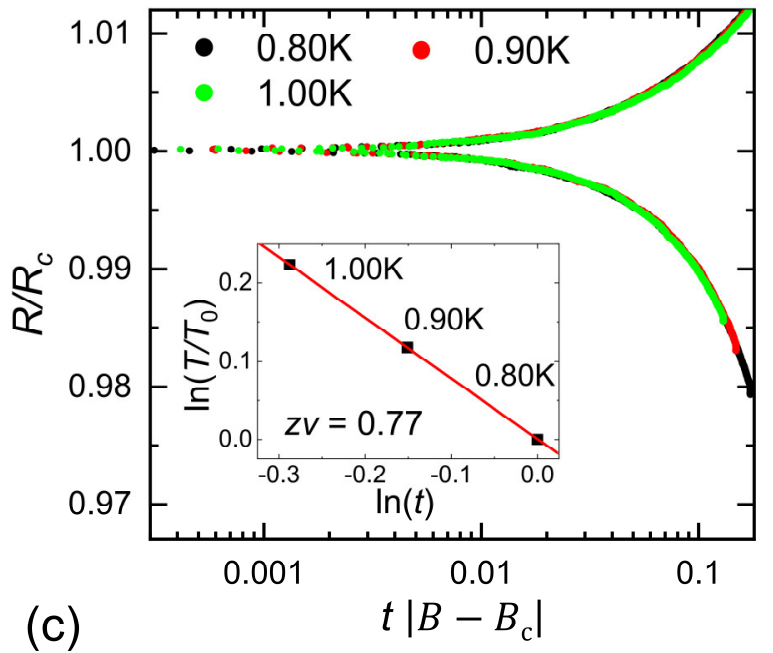

(b)

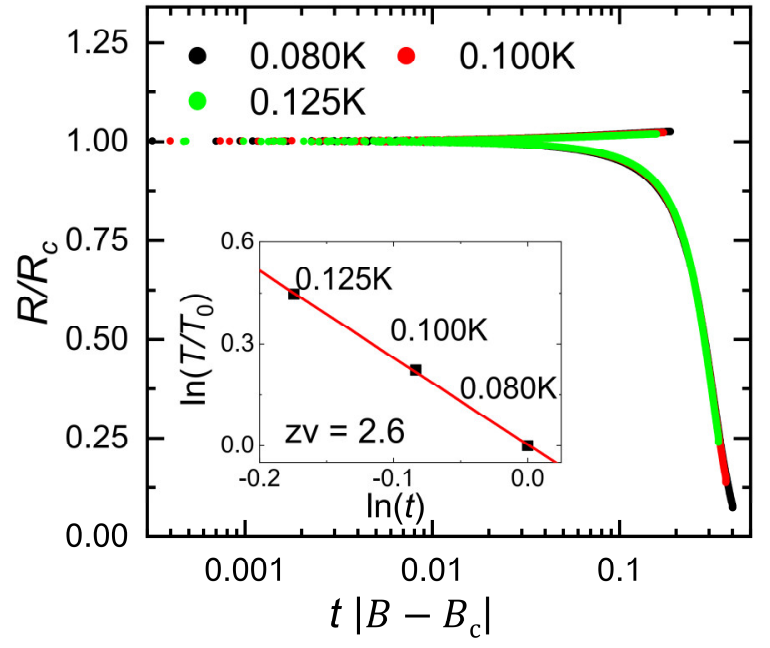

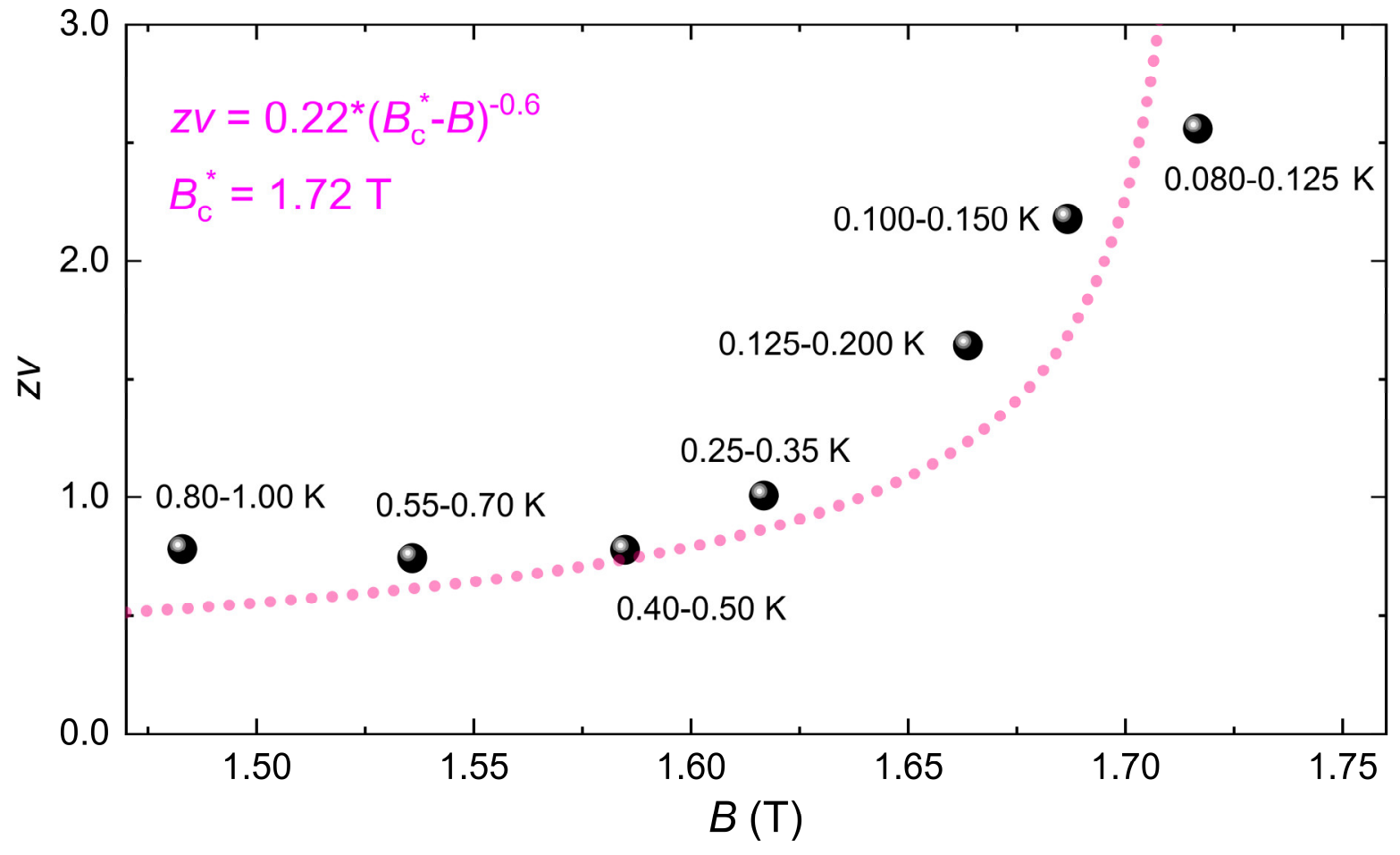

Figure 4. Experimental evidence of quantum Griffiths singularity at the $\mathrm{EuO} / \mathrm{KTaO}_{3}$ interface. (a-b) The scaling analysis of the interface superconductivity for two typical temperature regimes $(0.80 \mathrm{~K}-1.00 \mathrm{~K}$ and $0.080 \mathrm{~K}-0.125 \mathrm{~K})$. (c) The magnetic field dependence of dynamical critical exponent $(z v)$ in various temperature regimes. The purple dashed line represents the best fit based on the equation $z v=0.22 \times\left(B_{c}^{*}-B\right)^{-0.6}$. 


\section{Supplementary Materials for}

\section{Superconductor-metal quantum transition at the $\mathrm{EuO} / \mathrm{KTaO}_{3}$ interface}

Yang $\mathrm{Ma}^{1 \dagger}$, Jiasen $\mathrm{Niu}^{1 \dagger}$, Wenyu Xing ${ }^{1}$, Yunyan $\mathrm{Yao}^{1}$, Ranran Cai ${ }^{1}$, Jirong $\mathrm{Sun}^{2,3}, \mathrm{X} . \mathrm{C}$. $\mathrm{Xie}^{1,4,5}, \mathrm{Xi} \operatorname{Lin}^{1,4,5 *}$, Wei $\operatorname{Han}^{1 *}$

${ }^{1}$ International Center for Quantum Materials, School of Physics, Peking University, Beijing 100871, P. R. China

${ }^{2}$ Beijing National Laboratory for Condensed Matter Physics \& Institute of Physics, Chinese Academy of Sciences, Beijing 100190, P. R. China

${ }^{3}$ School of Physical Sciences, University of Chinese Academy of Sciences, Beijing 100049, P. R. China

${ }^{4}$ CAS Center for Excellence in Topological Quantum Computation, University of Chinese Academy of Sciences, Beijing 100190, P. R. China

${ }^{5}$ Beijing Academy of Quantum Information Sciences, Beijing 100193, P. R. China

†These authors contributed equally to the work

*Correspondence to: xilin@pku.edu.cn (X.L.) and weihan@pku.edu.cn (W.H.) 


\section{Supplementary Note 1. Estimation of the coherence length of the superconducting $\mathrm{EuO} / \mathrm{KTaO}_{3}$ interface.}

The coherence length $\left(\xi_{G L}\right)$ at absolute zero tempeature is calculated using the following equation,

$$
\xi_{G L}=\sqrt{\frac{\Phi_{0}}{2 \pi B_{\mathrm{C} \perp(T \rightarrow 0)}}},
$$

where $\Phi_{0}$ is the quantum flux, $B_{\mathrm{C} \perp(T \rightarrow 0)}$ is the critical perpendicular magnetic field at absolute zero tempeature. $B_{\mathrm{C} \perp}$ at each tempeature can be obtained form the half value of the normal resitance during the superconductor-metal transition. Based on the $B_{\mathrm{C} \perp}$ vs. $T$ curve (Fig. S2), the critical magnetic field at zero temperature is determined to be $\sim 1.51 \mathrm{~T}$, and the coherence length is calculated to be $\sim 14.8 \mathrm{~nm}$. 
(a)

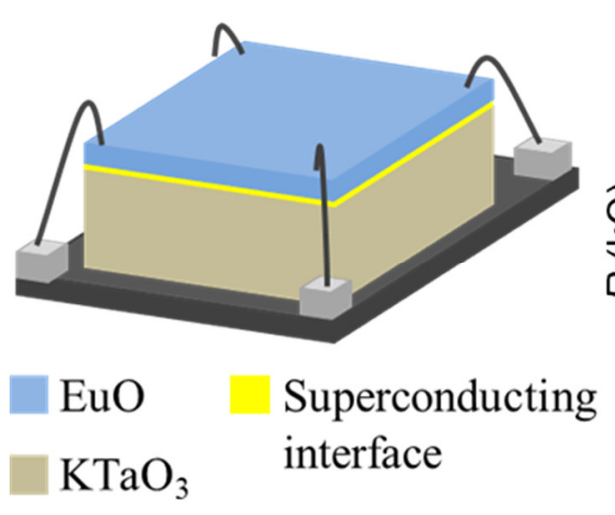

(b)

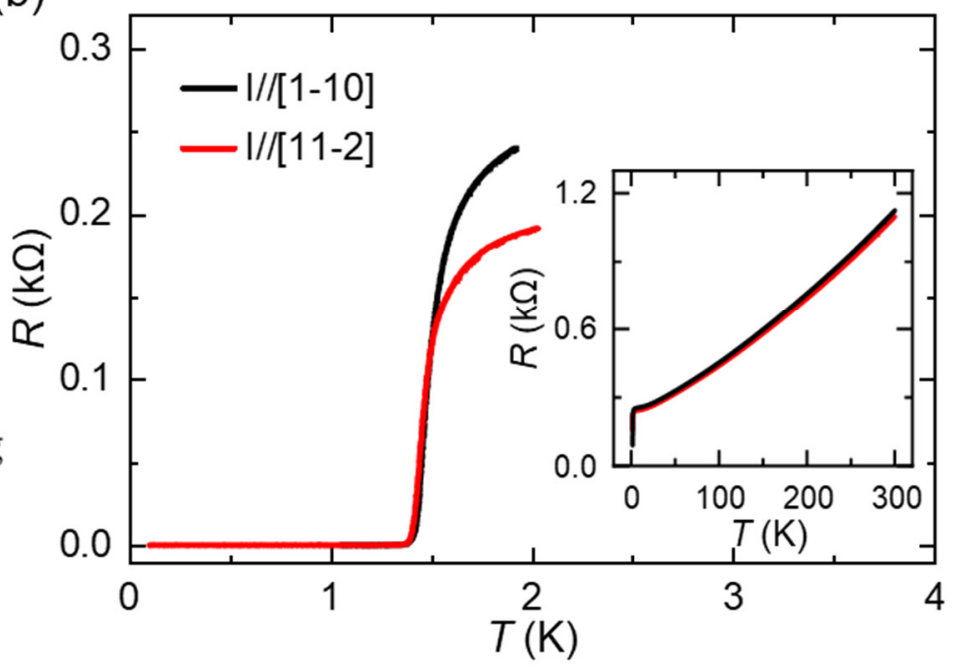

Figure S1. The transport properties of the $\mathrm{EuO} / \mathrm{KTaO}_{3}$ interface in the $\mathrm{VdP}$ geometry.

(a) The schematic illustration of the van der Pauw measurement geometry of the $\mathrm{EuO} / \mathrm{KTaO}_{3}$ interface. (b) The electrical measurement of the interface superconductivity with the current along the $\mathrm{KTaO}_{3}$ substrate's [1-10] and [11-2] directions. Inset: The measured resistance as a function of temperature from $300 \mathrm{~K}$ to $1.5 \mathrm{~K}$. 

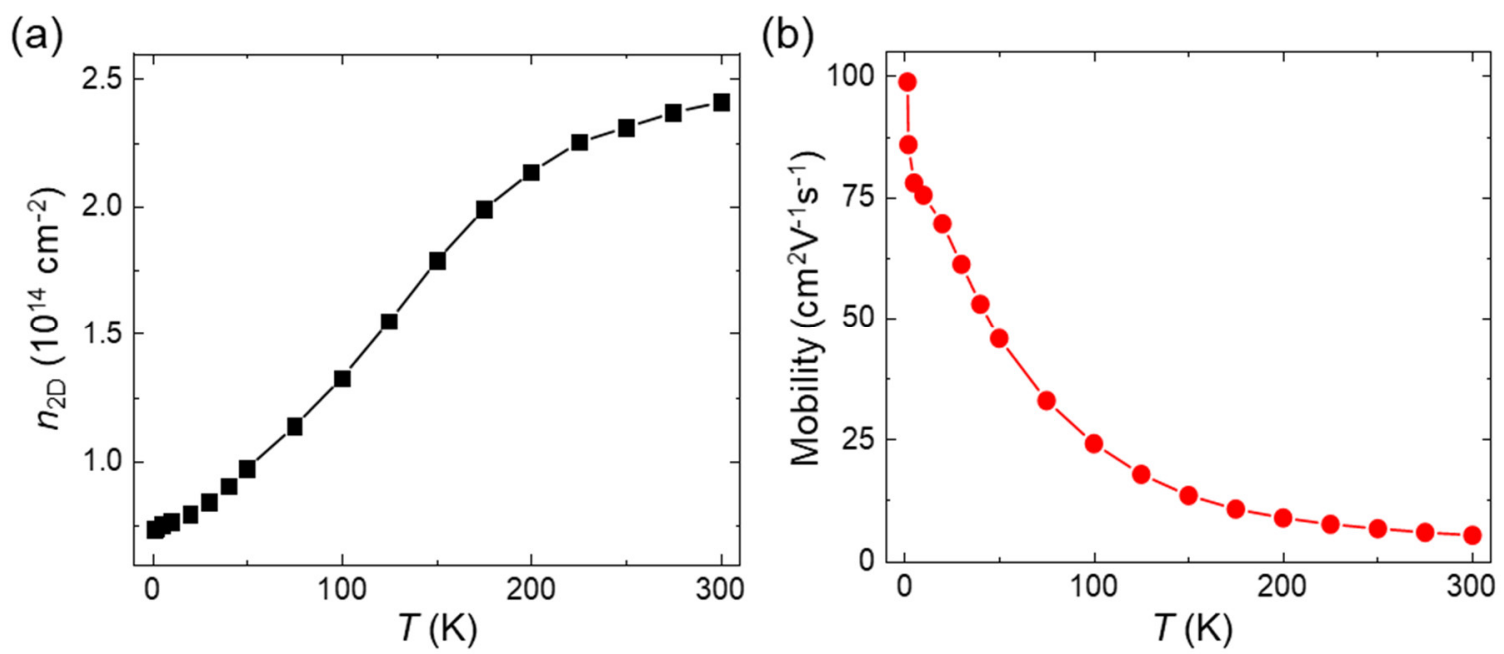

Figure S2. The electron transport properties of the $\mathrm{EuO} / \mathrm{KTaO}_{3}$ interface above $T_{\mathrm{C}}$. (a-b) The sheet carrier density and electron mobility as a function of temperature. 


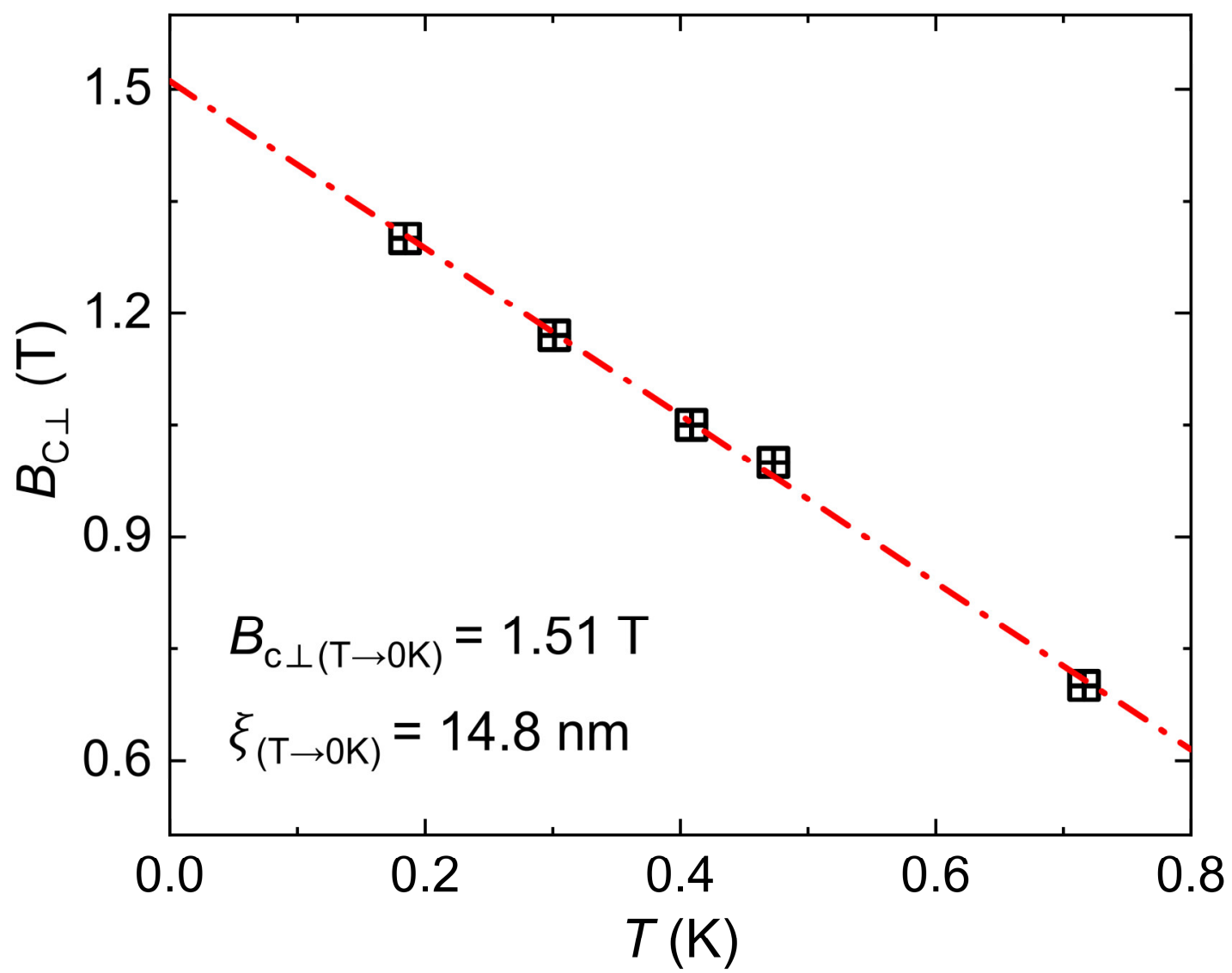

Figure S3. Temperature dependence of the critical perpendicular magnetic field of the $\mathrm{EuO} / \mathrm{KTaO}_{3}$ interface. The red line represents the best linear fit. 